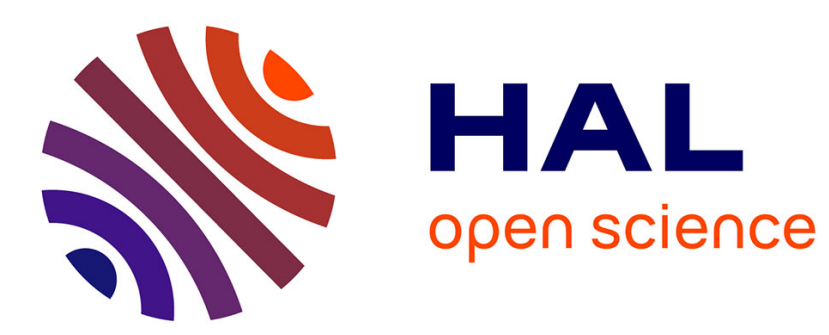

\title{
Transfer inside pairs of Pr3+ in LaF3 studied by up-conversion fluorescence
}

\author{
R. Buisson, J.C. Vial
}

\section{To cite this version:}

R. Buisson, J.C. Vial. Transfer inside pairs of Pr3+ in LaF3 studied by up-conversion fluorescence. Journal de Physique Lettres, 1981, 42 (5), pp.115-118. 10.1051/jphyslet:01981004205011500 . jpa00231885

\section{HAL Id: jpa-00231885 https://hal.science/jpa-00231885}

Submitted on 1 Jan 1981

HAL is a multi-disciplinary open access archive for the deposit and dissemination of scientific research documents, whether they are published or not. The documents may come from teaching and research institutions in France or abroad, or from public or private research centers.
L'archive ouverte pluridisciplinaire HAL, est destinée au dépôt et à la diffusion de documents scientifiques de niveau recherche, publiés ou non, émanant des établissements d'enseignement et de recherche français ou étrangers, des laboratoires publics ou privés. 


\title{
Transfer inside pairs of $\mathrm{Pr}^{3+}$ in $\mathrm{LaF}_{3}$ studied by up-conversion fluorescence
}

\author{
R. Buisson and J. C. Vial \\ Laboratoire de Spectrométrie Physique (*), Université Scientifique et Médicale de Grenoble, \\ B.P. 53 X, 38041 Grenoble Cedex, France
}

(Reçu le 22 décembre 1980, révisé le 19 janvier 1981, accepté le 19 janvier 1981)

\begin{abstract}
Résumé. - Ce travail présente des résultats expérimentaux montrant, pour la première fois, un transfert à l'intérieur d'une paire d'ions à la suite d'une excitation sélective par un laser pulsé. Ce transfert est étudié avec $\operatorname{Pr}^{3+}: \mathrm{LaF}_{3}$ en mesurant la dynamique d'une fluorescence de up-conversion. Les résultats montrent que deux mécanismes doivent être invoqués, selon la distance qui sépare les deux ions de chaque paire, pour expliquer les taux de transfert mesurés sur 11 paires différentes.
\end{abstract}

\begin{abstract}
Experimental results are reported which show for the first time a transfer inside pairs of ions after their selective excitation by a pulsed laser. This transfer is studied in $\operatorname{Pr}^{3+}: \mathrm{LaF}_{3}$ by monitoring the time dependence of an up-conversion fluorescence. The results show that two mechanisms must be considered, depending upon the distance between the ions of the pairs, to explain the transfer rates measured on 11 different pairs.
\end{abstract}

The study of energy transfer between identical ions in crystals has found new interest since the development of lasers and time resolved spectroscopy techniques. The observation of a transfer within the inhomogeneous width of optical lines [1, 2] has stimulated theoretical analysis to improve the models describing the transfer between randomly distributed ions $[3,4,5]$. These models, as well as the previous ones $[6,7]$, predict the time variation of the donor fluorescence which can be distinguished from that of acceptors by the frequency line narrowing techniques $[8,2]$. In these models, use is made of the " microscopic scale random broadening " hypothesis according to which the probability for a given distribution of dopant ions around a given one is the same for each one. This hypothesis permits to deduce by a statistical averaging the time variation of the donor fluorescence when a transfer mechanism has been postulated $[5,8]$.

A typical system to which the results of these calculations have been applied is $\operatorname{Pr}^{3+}: \mathrm{LaF}_{3}$ whose energy levels of interest are shown in figure 1.

Flach, Hamilton, Selzer and Yen $[2,8]$ have studied the transfer within the inhomogeneous width of the ${ }^{3} \mathrm{H}_{4}{ }^{-3} \mathrm{P}_{0}$ transition. With the highly concentrated crystals used $(5 \%$ and $20 \%)$, the linewidth is induced by the doping ions themselves acting as point defects. The microscopic broadening hypothesis is then valid

(*) Laboratoire associé au C.N.R.S.

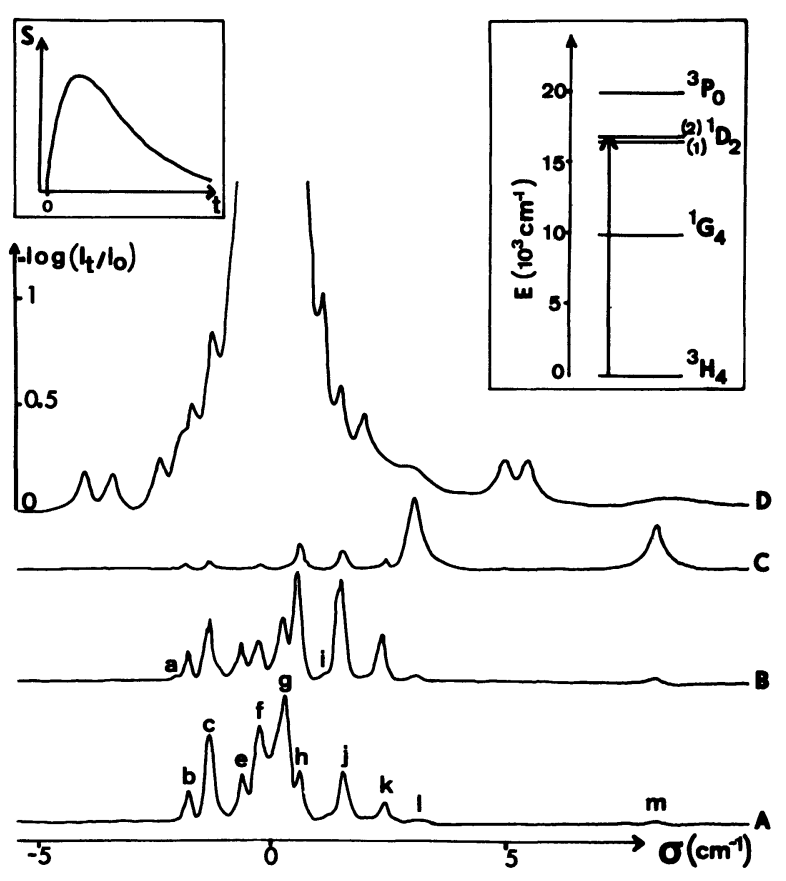

Fig. 1. - The three lower curves are excitation spectra obtained at $T=1.2 \mathrm{~K}$ with a $0.1 \%$ doped crystal by monitoring the ${ }^{3} \mathrm{P}_{6}$ fluorescence with a delay of $50 \mu \mathrm{s}$ (B), $3 \mu \mathrm{s}$ (C) (B and C are drawn with the same vertical scale), or by time-integrating this fluores cence for each laser pulse (A). Curve (D) is a high resolution absorption spectrum of a $17 \mathrm{~mm}$ thick $0.2 \%$ doped crystal. The origin of the energy scale is at the maximum of the ${ }^{3} \mathrm{H}_{4} \rightarrow{ }^{1} \mathrm{D}_{2}$ (1) absorption line. Small letters refer to the various lines of the excitation spectra. Left insert shows the qualitative time evolution of the ${ }^{3} P_{6}$ fluorescence. Right insert shows the energy levels of interest of $\mathrm{Pr}^{3+}: \mathrm{LaF}_{3}$ 
and the above models can indeed be used. It was thus found, after studying the influence of the concentration, that transfer is induced by dipole-dipole interaction [8].

With crystals of lower concentration, satellites of the optical lines have been observed and interpreted as due to pairs of ions [9], each one being associated with a class of pairs. In the more concentrated crystals, they were folded into the wings of the main line, but their existence has been inferred to explain some results [2]. With the low concentration crystals, the situation appears to be favourable for a study of the transfer between the ions of a pair, since their selective excitation is possible. In this paper, an experimental method is described which permits, for the first time, a direct observation of a transfer within 11 different classes of pairs of $\mathrm{Pr}^{3+}$ in $\mathrm{LaF}_{3}$.

The experimental assembly, previously described in detail [9], consists of a $\mathbf{N}_{2}$-laser which pumps a dye laser whose spectral width is less than $0.1 \mathrm{~cm}^{-1}$ owing to an intracavity Fabry-Pérot etalon. When the laser pulse has a wavelength corresponding to one of the pair lines seen on the high resolution absorption spectrum of figure 1 , it selectively excites ions of this class of pairs into the ${ }^{1} \mathrm{D}_{2}(2)$ level. These ions relax in a very short time $\left(\sim 10^{-9} \mathrm{~s}\right)$ to the ${ }^{1} \mathrm{D}_{2}(1)$ metastable level. When both ions of a pair are excited, a transfer occurs by which one ion gains energy and goes, after very fast relaxation, to the ${ }^{3} \mathrm{P}_{0}$ metastable level, while the other loses energy and goes to the lower excited level ${ }^{1} \mathrm{G}_{4}$. The former then emits an anti-Stokes (or up-conversion) fluorescence. Time resolved excitation spectra have been obtained by monitoring this fluorescence as a function of the laser wavelength through a box car integrator having its gate at a fixed delay after the laser pulse. The time variation of this fluorescence for a fixed excitation wavelength has also been studied with a multichannel analyser.

Before presenting the results, it is important to point out some specific features of the experimental method. The excitation is similar to that used in the experiments of site selection spectroscopy, either within the inhomogeneous lines [2] or among the various crystal sites occupied by the impurity ions $[10,11]$. In fact, in this work the pairs are selected instead of isolated ions and transfer within a pair is studied instead of transfer between the various classes of (supposed) isolated ions. The major advantage of the method lies in its double selectivity. Indeed, the usual spectral selectivity of the excitation is followed by a selectivity at the detection : only ions having participated in the transfer emit the anti-Stokes fluorescence. In other words, both donors and acceptors are selected whereas only donors are usually selected. This is important both conceptually and experimentally. Indeed, the transfer observed is " purer " since it occurs between two well defined ions. Experimentally, instead of monitoring the fluorescence of donors and extracting the information on the transfer from the non-exponential part of the decay, one observes a signal due only to the transfer within a pair.

In figure 1 is drawn a high resolution absorption spectrum, obtained at $T=1.2 \mathrm{~K}$ with a $2 \mathrm{~m}$ SOPRA monochromator having a resolution of $0.03 \mathrm{~cm}^{-1}$. This spectrum shows the satellite lines of the ${ }^{3} \mathrm{H}_{4} \rightarrow{ }^{1} \mathrm{D}_{2}$ (2) transition. Time resolved excitation spectra obtained at $T=1.2 \mathrm{~K}$ by monitoring the ${ }^{3} \mathrm{P}_{0}$ fluorescence through a wide band filter to eliminate the ${ }^{1} D_{2}(1)$ fluorescence are also given as well as a " time integrated " excitation spectrum obtained by monitoring the total energy of the ${ }^{3} \mathrm{P}_{0}$ fluorescence induced by a laser pulse. These spectra show two important qualitative features. Firstly, the comparison between absorption and integrated excitation spectra shows a dramatic variation of the quantum yield for the up-conversion transfer. Small pair-lines which absorb a few $\%$ of the laser light produce much more integrated blue fluorescence than the central part of the main line for which the transmission is well below $1 \%$. Secondly, the dynamics of the ${ }^{3} \mathrm{P}_{0}$ fluorescence strongly change with the delay time of the box car, as can be seen from the modification of the shape of the spectrum. This change is best illustrated by a direct observation of the time variation of the fluorescence as shown qualitatively in one insert of figure 1. This time variation can be established from the interpretation of the up-conversion transfer. Let $N_{\mathrm{d}}$ be the number of pairs, among a given class, whose two ions have been excited by the laser pulse. The time evolution of $N_{\mathrm{d}}$ is given by

$$
\dot{N}_{\mathrm{d}}=-2 W_{2} N_{\mathrm{d}}-W_{\mathrm{t}} N_{\mathrm{d}}
$$

where $W_{\mathrm{t}}$ is the transfer rate and $W_{2}$ the radiative decay rate of individual ions from the ${ }^{1} D_{2}(1)$ level. The factor 2 appears because a doubly excited pair is destroyed as soon as one of its ions de-excites. The number $N_{3}$ of ions excited into the ${ }^{3} \mathrm{P}_{0}$ level by the transfer varies as

$$
\dot{N}_{3}=W_{\mathrm{t}} N_{\mathrm{d}}-W_{3} N_{3}
$$

where $W_{3}$ is the decay rate from ${ }^{3} \mathrm{P}_{0}$. The solution of equations (1) and (2) is straightforward and leads for the population $N_{3}$, to which the fluorescence is proportional, to the value

$N_{3}=\frac{N_{\mathrm{d}}^{0} W_{\mathrm{t}}}{W_{3}-2 W_{2}-W_{\mathrm{t}}}\left(\mathrm{e}^{-\left(2 W_{2}+W_{\mathrm{t}}\right) t}-\mathrm{e}^{-W_{3} t}\right)$

where $N_{\mathrm{d}}^{0}$ is the number of doubly excited pairs just after the laser pulse. Whatever the $W_{i}$ values, the variation of $N_{3}$ corresponds to that observed (see the insert in figure 1), the decay at long time being associated with the slower of the two rates $W_{3}$ or (2 $\left.W_{2}+W_{t}\right)$.

For each of the 13 lines visible on the spectra, we have fitted the ${ }^{3} \mathrm{P}_{0}$ fluorescence signal given by a 
1024 points multichannel analyser with equation

$$
S=A\left(\mathrm{e}^{-W_{\mathrm{d}} t}-\mathrm{e}^{-W_{\mathrm{r}} t}\right)
$$

where $W_{\mathrm{r}}$ and $W_{\mathrm{d}}$ are the observed rates for the rise and the decay of the fluorescence. For most of the lines, the fitting was obtained with good precision (better than $5 \%$ ), thus validiting the model. The results are given in table I for two different concentrations : $0.02 \%$ and $0.1 \%$. They show that the rates are essentially equal for these two concentrations (they change slightly more for $0.2 \%$ samples), indicating that transfers inside well defined and quasi-isolated pairs are observed. $W_{\mathrm{d}}$, as well as $W_{\mathrm{r}}$, can be either ( $2 W_{2}+W_{\mathrm{t}}$ ) or $W_{3}$. Ignoring pairs (f) and (g) as explained below, the correspondence

$$
W_{\mathrm{d}} \leftrightarrow\left(2 W_{2}+W_{\mathrm{t}}\right) \text { and } W_{\mathrm{r}} \leftrightarrow W_{3}
$$

have been made for pairs (a) to (k) consisting of not too near ions (since associated with lines not too far from the central line). Values of $W_{3}$ and $W_{t}$, deduced using $W_{2}=2 \mathrm{~ms}^{-1}$ [12] are given for these pairs in table I for the $0.02 \%$ sample for which the pairs are better " isolated " one from the others. For pairs (l) and (m), the values of $W_{3}$ and $W_{\mathrm{t}}$ given in table $\mathrm{I}$ have been obtained by making the correspondences $W_{3} \leftrightarrow W_{\mathrm{d}}$ and $\left(2 W_{2}+W_{\mathrm{t}}\right) \leftrightarrow W_{\mathrm{r}}$. This point is now discussed. It is first pointed out that the $W_{3}$ thus obtained is, for all the pairs, not strongly different from the radiative rate $\left(W_{3 \mathrm{R}}=21 \mathrm{~ms}^{-1}\right.$ [12]). The reduction of the lifetime by non radiative mechanisms appears thus to be small, a result which could be surprising since the known cross relaxation between two nearby ions [13] should have a large efficiency. But one must remember that only ions having participated in the up-conversion transfer has put ion $A$ into the ${ }^{3} \mathrm{P}_{0}$ level and ion $B$ into the ${ }^{1} \mathrm{G}_{4}$ level. The lifetime of this later level is estimated to $1 \mathrm{~ms}$ (a $300 \mathrm{~s}^{-1}$ radiative decay rate has been calculated by Weber [12] and a multiphonon decay rate of the same order of magnitude can be estimated from data presented by Reisfeld and Jorgensen [14]). That implies a low efficiency of the above cross relaxation which needs ion $B$ in the ground state or in a low excited state. The weak increase of the effective $W_{3}$ observed is thus compatible with this analysis whereas the large values of $W_{3}$ for pairs (l) and (m) obtained by making the correspondences $W_{3} \leftrightarrow W_{\mathrm{r}}$ and $\left(2 W_{2}+W_{\mathrm{t}}\right) \leftrightarrow W_{\mathrm{d}}$ $\left(W_{3}=1400\right.$ and $1800 \mathrm{~ms}^{-1}$ respectively for these pairs) are impossible to explain.

From the structure of $\mathrm{LaF}_{3}$ [15], it is easy to calculate the distance between the two ions of the various possible pairs. Since the transfer is known to be induced by the dipole-dipole interaction [8], the transfer probability $W_{\mathrm{t}}$ must change as $r^{-6}$ if the changes of the angular part of the dipole-dipole interaction are ignored. Referring by $p$, to the pair made of one ion and one of its $p$ th neighbours, one finds that $r$ varies from $4.15 \AA$ for $p=1$ to $10.3 \AA$ for $p=11$ and thus that the ratio $W_{\mathrm{t}}(p) / W_{\mathrm{t}}(11)$ takes the following values for $p=11$ to $p=1: 1-1.5-1.8-3.3$ 3.7-7.6-7.8-8.7-24-170-230. A comparison of this sequence with the following one : 1-1.3-2.3-5.2-11.312-13.5-14.4-15.1-1 600-2 100, obtained by a normalization with respect to the smallest of the $W_{\mathrm{t}}$ given in table I, shows a qualitative agreement for the 9 first numbers and a strong disagreement for the two last. Thus, the dipole-dipole interaction appears indeed as the transfer mechanism for most of the pairs, the differences in the variations along these sequences being due to its angular part. However, for pairs (l) and (m), it seems difficult to invoke this angular part to explain the disagreement. Instead, a mechanism whose variation with the distance between the ions of a pair is faster that $\left(r^{-6}\right)$ must be considered. Watts [16] has shown, by a numerical analysis of the various multipolar terms for $\mathrm{Yb}^{3+}-\mathrm{Er}^{3+}$ transfer, that quadrupolequadrupole interaction dominates for pairs separated by less than $7 \AA$ (in $\mathrm{LaF}_{3}, r=6.05 \AA$ for $p=3$ and $r=7.2 \AA$ for $p=4$ ). Although our results do not give the active mechanism for the two pairs made of nearest and next nearest-neighbours, they directly show, for the first time, that indeed two mechanisms can be active for the same system.

At the very centre of the main line the observed dynamics are not compatible with equation (3). This

Table I.

$\begin{array}{clllllllllllllr}\begin{array}{c}\text { Transition } \\ \text { probability }\end{array} & \begin{array}{c}x \\ (\%)\end{array} & \mathrm{a} & \mathrm{b} & \mathrm{c} & \mathrm{d} & \mathrm{e} & \mathrm{f} & \mathrm{g} & \mathrm{h} & \mathrm{i} & \mathrm{j} & \mathrm{k} & \mathrm{l} & \mathrm{m} \\ - & - & - & - & - & - & - & - & - & - & - & - & - & - & - \\ W_{\mathrm{d}} & 0.02 & 14.3 & 8.5 & 6.0 & 16.4 & 5.1 & 3.4 & 4.4 & 17.0 & 4.9 & 15.6 & 13.7 & 65 & 59 \\ & 0.1 & 15.0 & 8.8 & 6.2 & 14.0 & 5.0 & 4.1 & 4.6 & 14.4 & 5.0 & 15.8 & 13.3 & 61 & 55 \\ W_{\mathrm{r}} & 0.02 & 44 & 23 & 25 & 34 & 25 & 25 & 23 & 61 & 38 & 33 & 31 & 1400 & 1800 \\ W_{\mathrm{t}} & 0.1 & 48 & 25 & 28 & 42 & 28 & 22 & 27 & 81 & 64 & 34 & 34 & 1200 & 1700 \\ W_{3} & 0.02 & 10.3 & 4.5 & 2.0 & 12.4 & 1.1 & - & - & 13.0 & 0.86 & 11.6 & 9.7 & 1400 & 1800 \\ & 0.02 & 44 & 23 & 25 & 34 & 25 & - & - & 61 & 38 & 33 & 31 & 65 & 59\end{array}$

Lines (1), (2), (3), (4) give the values of $W_{\mathrm{d}}$ and $W_{\mathrm{r}}\left(\right.$ in ms $^{-1}$ ) obtained by fitting the observed ${ }^{3} \mathrm{P}_{0}$ fluorescence with equation (4) for the 13 satellites of the spectra given in figure 1 and for two $\operatorname{Pr}^{3+}$ concentrations $x$. Lines (5) and (6) give the values of $W_{t}$ and $W_{3}$ (in $\mathrm{ms}^{-1}$ ) deduced as explained in the text for $x=0.02$. 
equation shows that when $W_{\mathrm{t}} \rightarrow 0$ (which occurs for weakly coupled pairs whose satellite lines are near the centre) the rising rate $W_{\mathrm{r}}$ of the fluorescence must become $W_{3} \sim 21 \mathrm{~ms}^{-1}$ and the decay rate $2 W_{2} \sim 4 \mathrm{~ms}^{-1}$, whereas experiments give smaller and concentration dependent rising rate. This could be an indication of a diffusion of the excitation but other experiments and further analysis are necessary for a confirmation. This unexplained behaviour of the strong central line perturbs the dynamics of the nearly lines (f) and (g). This is the reason why these lines have been excluded in the above analysis.

We have reported here the first direct study of an energy transfer within well defined pairs whereas only the average effect of transfer, within all classes of pairs, are usually reported. This was made possible by using an experimental method by which both donor and acceptor ions are selected. The results confirm that dipole-dipole interaction is responsible for the transfer. The method seems promising to study the transfer in the intermediate situation for which the inhomogeneous broadening is only partly due to random causes making invalid the microscopic broadening hypothesis. The method can also be used to study the neighbourhood of an ion. For instance, it has been possible to distinguish between pairs of ions which mutually perturb their energy levels in the same manner and pairs of ions which do not. A forthcoming paper will present these results which explain the absence, in the excitation spectra of figure 1 , of lines associated with some absorption satellites whereas they were previously observed [9] when using a laser of larger spectral width.

Acknowledgments. - It is a pleasure for the authors to thank F. Madéore for his important contribution to the achievement of the performances of the laser, M. Poirier for his collaboration in part of this work and R. Romestain and Le Si Dang for very stimulating discussions.

\section{References}

[1] Flash, R., Hamilton, D. S., Selzer, P. M., Yen, W. M., Phys. Rev. Lett. 35 (1975) 1034.

[2] Flash, R., Hamilton, D. S., Selzer, P. M., Yen, W. M., Phys. Rev. B 15 (1977) 1248.

[3] Holstein, T., Lyo, S. K., Orbach, R., Phys. Rev. B 15 (1977) 4693.

[4] Holstein, T., Lyo, S. K., Orbach, R., Phys. Rev. B 16 (1977) 934.

[5] Huber, D. L., Hamilton, D. S., Barnett, B., Phys. Rev. B 16 (1977) 4642.

[6] Dexter, D. L., J. Chem. Phys. 21 (1953) 836.

[7] Inokuti, M., Hirayama, F., J. Chem. Phys. 43 (1965) 1978.

[8] Selzer, P. M., Hamilton, D. S., Flash, R., Yen, W. M., J. Lumin. 12/13 (1976) 737.
[9] Vial, J. C., Buisson, R., Madeore, F., Poirier, M., J. Physique 40 (1979) 913.

[10] Merkle, L. D., Powell, R. C., Phys. Rev. B 20 (1979) 75.

[11] Kliava, J., Evesque, P., Duran, J., J. Phys. C : Solid State Phys. 11 (1978) 3357.

[12] Weber, M. J., J. Chem. Phys. 48 (1968) 4774.

[13] Brown, M. R., Whiting, J. S. S., Shand, W. A., J. Chem. Phys. 43 (1965) 1.

[14] ReISFELD, R., JORGENSEN, C. K., Lasers and excited states of Rare Earths (Springer-Verlag, Berlin, Heidelberg) 1977. [15] von Mansmann, M., Z. Kristall. 122 (1965) 375.

[16] Watts, R. K., in Optical Properties of ions in solids, edited by Di Bartolo, B. (Plenum, New York) 1975. 